\title{
Evaluation of the Antimicrobial Effect of Mineral Trioxide Aggregate Mixed with Fluorohydroxyapatite against E. faecalis In Vitro
}

\author{
Behnam Bolhari $\mathbb{D}^{1},{ }^{1}$ Aidin Sooratgar $\mathbb{D}^{2},{ }^{2}$ Maryam Pourhajibagher $\left(\mathbb{D},{ }^{3}\right.$ \\ Nazanin Chitsaz $\mathbb{D}^{1},{ }^{1}$ and Iman Hamraz $\mathbb{D}^{4}$ \\ ${ }^{1}$ Department of Endodontics, School of Dentistry, Tehran University of Medical Sciences (TUMS), Tehran, Iran \\ ${ }^{2}$ Department of Endodontics, Tehran University of Medical Sciences (TUMS), International Campus, Tehran, Iran \\ ${ }^{3}$ Dental Research Center, Dentistry Research Institute, Tehran University of Medical Sciences (TUMS), Tehran, Iran \\ ${ }^{4}$ Department of Biostatistics, Faculty of Medicine, Arak University of Medical Sciences, Arak, Iran
}

Correspondence should be addressed to Nazanin Chitsaz; chitsaz.nazanin11@yahoo.com

Received 15 September 2021; Revised 8 November 2021; Accepted 10 November 2021; Published 24 November 2021

Academic Editor: Isabel Del Hierro

Copyright (C) 2021 Behnam Bolhari et al. This is an open access article distributed under the Creative Commons Attribution License, which permits unrestricted use, distribution, and reproduction in any medium, provided the original work is properly cited.

\begin{abstract}
Enterococcus faecalis is the dominant microorganism in chronic apical periodontitis. It is more resistant to local antiseptic agents than other endodontic microorganisms. Currently, mineral trioxide aggregate (MTA) is considered as an ideal material in many endodontic procedures. Some studies have shown that MTA has good antibacterial activity against E. faecalis. However, some studies have investigated the effect of incorporating some materials into MTA on its antibacterial activity against $E$. faecalis. No study has evaluated the effect of incorporating fluorohydroxyapatite nanoparticles (nano-FHA) on the antimicrobial activity of MTA. Therefore, the present study evaluated the antimicrobial effect of MTA mixed with nano-FHA on E. faecalis in vitro. The study was carried out on 18 samples in three groups: pure MTA, MTA mixed with $10 \mathrm{wt} \%$ of nano-FHA, and MTA mixed with 15 wt $\%$ of nan-FHA. The effect of nano-FHA on the antibacterial activity of MTA on E. faecalis was evaluated by evaluating the growth inhibition zone around each sample. The antimicrobial effect of samples on inhibiting E. faecalis biofilm formation and inhibiting microbial growth of $E$. faecalis in the planktonic phase was evaluated by disk agar diffusion (DAD), biofilm inhibition assay (BIA), and direct contact assay (DCA) tests, respectively. All the above tests were analyzed after 24 and 72 hours. Factorial designs were used for statistical analyses. Tukey tests were used for two-by-two comparisons. All the statistical analyses were carried out with SPSS 26. DAD results showed no formation of the growth inhibition zone in all the samples after 24 and 72 hours. The microbial colony counts in the BIA and DCA tests in the groups modified with FHA nanoparticles were significantly lower than the pure MTA group $(P<0.05)$. The microbial colony counts increased in all the groups over time $(P<0.05)$. Incorporating nano-FHA into MTA improved the antimicrobial activity of MTA against E. faecalis compared to pure MTA. The highest antimicrobial activity was achieved after incorporating $15 \mathrm{wt} \%$ of nano-FHA into MTA at the 72-hour interval.
\end{abstract}

\section{Introduction}

The main reasons for pulpal and periapical diseases and failure of endodontic treatments are microorganisms $[1,2]$. Therefore, eliminating microorganisms by instrumentation, irrigation, and intracanal medications during root canal treatment is necessary [3]. However, it is not possible to completely clean root canals from bacteria and their products due to the complexity of the root canal system. Therefore, proper obturation of the entire root canals or the perforation area has a vital role in preventing reinfection by residual microorganisms due to the antimicrobial activity of sealing materials $[4,5]$. MTA is composed of $80 \%$ Portland cement and $20 \%$ bismuth oxide (radiopacifier). The cement is made up of calcium, silicon, and aluminium. The main constituent phases are tricalcium and dicalcium silicate and 
tricalcium aluminate. There are gray and white forms of MTA. The phase tetracalcium aluminoferrite is absent in white MTA [6]. Currently, MTA is considered as an ideal material in many endodontic processes, including pulp capping, pulpotomy, apexogenesis/apexification, root resorption, lateral or furcal perforation repair, and retrograde obturation [7] because this material induces the growth of cementum and PDL, resulting in proper periodontal healing [8]. However, MTA has some inherent disadvantages, including difficult handling, long setting time, and discoloration potential [9]. Since the introduction of ProRoot MTA in 1998, new products have been produced based on MTA, including MTA Angelus and Endocem MTA, to overcome these deficiencies by modifying the composition or concentration of each material [7]. MTA Angelus (Angelus, Londrina, PR, Brazil) is a biocompatible material produced for bone repair procedures and has been compared with Portland cement due to similarities in their chemical structure and tissue response $[10,11]$.

Recently, several studies have evaluated the effect of MTA on microorganisms related to endodontic diseases [10, 12, 13]. Enterococci, Actinomycetes, Propionibacterium, yeasts, and Streptococci are some of the microorganisms isolated from the infected root canals [14]. However, many clinicians believe that E. faecalis is the dominant microorganism in chronic apical periodontitis (retreatment cases) $[15,16]$. This microorganism is a Gram-positive [1], facultative anaerobic, bacterial species [17] that can survive without water and nutrients for several months [18]. It is also more resistant to local disinfectants than other endodontic microorganisms [19].

Some studies, including that by Morgental et al. (2011), have shown that MTA does not exhibit significant antibacterial activity against E. faecalis after setting [20]. Therefore, several studies have evaluated the effect of incorporating some materials, including $2 \%$ chlorhexidine (CHX) gluconate, tetracycline powder, and calcium hydroxide, on the antibacterial activity of MTA against E. faecalis, concluding that incorporating CHX into MTA increased its antimicrobial activity [14].

Hydroxyapatite (HA) is a mineral agent with a chemical structure of $\mathrm{Ca}_{10}\left(\mathrm{PO}_{4}\right)_{6}(\mathrm{OH})_{2}$ that comprises $60-70 \%$ of the mineral matrix of the bone and has high bioactivity and biocompatibility $[21,22]$. This material has many applications in biomedicine, especially in orthopedics and dentistry [23]. Incorporating fluorine into hydroxyapatite results in the synthesis of fluorohydroxyapatite (FHA: $\left.\mathrm{Ca}_{5}\left(\mathrm{PO}_{4}\right)_{3}(\mathrm{OH})_{1-\mathrm{x}} \mathrm{F}_{\mathrm{x}}\right)$, which is more stable chemically than hydroxyapatite, with low solubility [3]. Some studies have even shown that FHA has higher antimicrobial activity than pure HA [24].

Promising characteristics of FHA have made it a good material in orthopedics and dentistry [25]. However, limited reports are available on incorporating this material into other commonly used materials in dentistry, and no study is available on the effect of this material on the antimicrobial activity of MTA. Therefore, the present study aimed to evaluate the antimicrobial activity of MTA mixed with fluorohydroxyapatite against $E$. faecalis in vitro.

\section{Materials and Methods}

2.1. The Synthesis of Fluorohydroxyapatite (Nano-FHA). Four-water calcium nitrate solution was prepared with $0.3 \mathrm{M}$ concentration and placed on a magnetic mixer. The electrode of the $\mathrm{pH}$ meter (WTW, Germany) was placed within the reaction container to measure the $\mathrm{pH}$ of the container continuously. Then, a $1 \mathrm{M}$ solution of sodium hydroxide was added to the solution until the $\mathrm{pH}$ was stabilized between 10 and 11. Subsequently, $0.18 \mathrm{M}$ ammonium dihydrogen phosphate and $0.18 \mathrm{M}$ sodium fluoride were added to the calcium nitrate solution dropwise. During the reaction, the $\mathrm{pH}$ was kept between 10 and 11 by adding $\mathrm{NaOH}$.

To separate fluorohydroxyapatite, the resulting solution was cleared through a filter, and the remaining liquid was transferred to a centrifuge container. The liquid was washed with water and finally with acetone after several times of centrifugation. The gels achieved from the centrifugation process were dried by freeze-drying. The dried powder was sintered in an oven at $600^{\circ} \mathrm{C}$ for 6 hours. The heat increase rate in the oven was $2^{\circ} \mathrm{C} / \mathrm{min}[26]$.

2.2. Preparation of Samples. The pilot was done to determine the percentages of adding FHA to MTA. Adding percentages lower than $10 \%$ did not give any special properties to the material. Adding percentages greater than $15 \%$ made difficult handling of the material.

To prepare the test groups, the commercial MTA powder was mixed with 10 and $15 \mathrm{wt} \%$ of nano-FHA as follows: nano-FHA and commercial MTA powders were weighed with an accurate weighing machine and divided into equal parts. Then, each equal pat of nano-FHA powder (for one group with a $10 \mathrm{wt} \%$ of the final powder and for one group with a $15 \mathrm{wt} \%$ of the final powder) was manually mixed with MTA Angelus powder for three minutes, followed by mixing in an amalgamator (Golden Amalgamator, China), to achieve a uniform distribution of particles. Therefore, three groups were prepared as follows (Figure 1):
(1) MTA
(2) MTA + FHA (10 wt $\%)$
(3) MTA + FHA (15 wt\%)

2.3. Antimicrobial Tests. The effect of nano-FHA on the antimicrobial activity of MTA against E. faecalis was evaluated by evaluating the growth inhibition zone around each sample. In addition, the antimicrobial effect of the samples on inhibiting E. faecalis biofilm formation and growth inhibition of E. faecalis in the planktonic phase was evaluated with DAD, BIA, and DCA tests. All the above tests were analyzed after 24 and 72 hours.

2.4. Disk AgarDiffusion Test. An $0.5 \mathrm{McF}$ arland suspension $\left(1.5 \times 10^{8} \mathrm{CFU} / \mathrm{mL}\right)$ was prepared from $E$. faecalis bacterial species in the BHI (brain heart infusion) broth (Merck, Darmstadt, Germany) and seeded on the BHI agar 


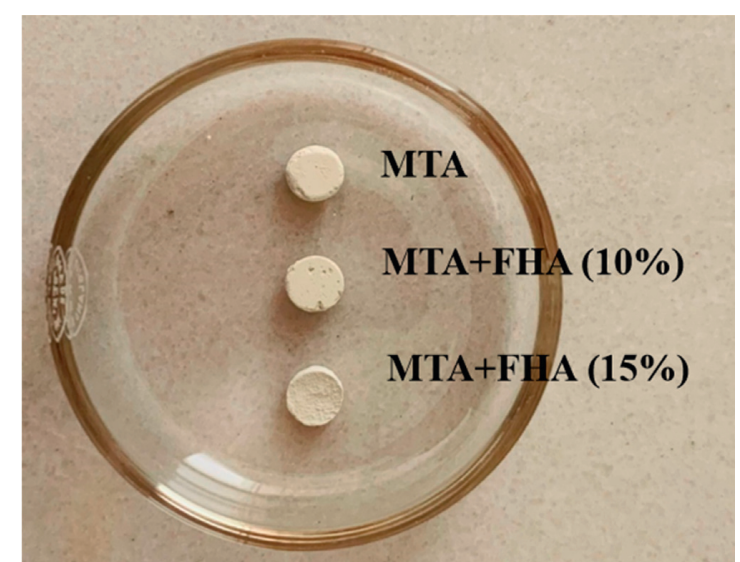

FIGURE 1: The samples were prepared in three study groups.

medium [27]. After the culturing procedure, the prepared disks were placed on the surface of the culture medium inoculated with microorganisms at a distance of $2 \mathrm{~mm}$ from each other, and the plates were incubated at $37^{\circ} \mathrm{C}$ for 24 hours [28]. The results were reported by measuring the diameters of the growth inhibition zones with a ruler [29].

2.5. Biofilm Inhibition Test. To carry out this test, first, the disks containing different concentrations of nano-FHA were placed in 48-well microplates. Then, $1 \mathrm{~mL}$ of the microbial suspension at $0.5 \mathrm{McF}$ arland concentration $\left(1.5 \times 10^{8} \mathrm{CFU} /\right.$ $\mathrm{mL}$ ) was added to each well. Subsequently, the 48-well microplates were incubated at $37^{\circ} \mathrm{C}$ for 24 and 72 hours [28]. After these time intervals, each disk was rinsed in tubes containing $1 \mathrm{~mL}$ of sterile normal saline solution for 1 minute. A sonicator was used to detach microbial biofilms formed on the disk surfaces. Then, the microbial suspensions achieved were diluted and cultured in a BHI agar medium. The microbial colony counts were determined based on previous studies [30].

2.6. Direct Contact Assay. To carry out this test, the disks with different concentrations were placed in tubes containing $500 \mu \mathrm{L}$ of the microbial suspension with a concentration of $1.5 \times 10^{8} \mathrm{CFU} / \mathrm{mL}$ and incubated at $37^{\circ} \mathrm{C}$ for 24 and 72 hours. After these time intervals, $10 \mu \mathrm{L}$ of the content of each tube was transferred into the BHI agar medium and spread-cultured on the surface of the culture mediums. The colony counts were determined similar to previous studies and reported in $\mathrm{CFU} / \mathrm{mm}^{2}$ [28].

2.7. Statistical Analysis. The descriptive data of the study were reported using descriptive statistics, including means, minimums, maximums, and standard deviations. Factorial designs were used to compare microbial tests, and the tests were reported three times considering the factors of time and the type of the microbial test. Tukey tests were used for twoby-two comparisons. SPSS 26 was used for all the statistical analyses.

\section{Results}

3.1. The Results of the Disk Agar Diffusion Antimicrobial Test. This test showed the absence of growth inhibition zones in all the samples after 24 hours (Figure 2).

\subsection{The Results of Biofilm Inhibition and Direct Contact Assay} Antimicrobial Tests. Factorial designs were used to evaluate the antimicrobial effect of MTA-FHA on E. faecalis at 24and 72-hour intervals. Table 1 presents the descriptive statistics (colony counts) in the study groups at the above intervals based on DCA and BIA antimicrobial tests.

According to Table 1, the DCA test showed a decrease in microbial biofilm formation on the disk surfaces with an increase in the percentage of FHA incorporated into MTA. After 24 hours, the microbial biofilm in the MTA group modified with $10 \%$ FHA decreased $24.47 \%$ compared to the pure MTA group $(P<0.05)$; in the MTA group modified with 15\% FHA, it decreased $45.57 \%$ compared to the pure MTA group $(P<0.05)$. After 72 hours, the microbial biofilm in the MTA group modified with $10 \%$ FHA decreased $18.49 \%$ compared to the pure MTA group $(P<0.05)$; in the MTA group modified with $15 \%$ FHA, it decreased $43.7 \%$ compared to the pure MTA group $(P<0.05)$.

In all the study groups, biofilm formation increased over time after 72 hours compared to the 24-hour interval (the pure MTA group: $32.2 \%$ increase, the MTA-10\% FHA group: $42.67 \%$ increase, and the MTA-15\% FHA group: $36.74 \%$ increase $)(P<0.05)$.

According to Table 2, the results of the BIA test were similar to those of the DCA test: an increase in the percentage of FHA incorporated into MTA decreased microbial biofilm formation $(P<0.05)$ (at both 24 - and 72-hour intervals). The microbial biofilm formation increased after 72 hours compared to the 24-hour interval in all the study groups $(P<0.05)$.

Table 3 presents the results of two-by-two comparisons of the groups with post hoc Tukey tests. Based on the results, in the DCA test, the greatest differences were observed between the MTA and MTA-15\% FHA groups $(P<0.001)$, followed by the MTA-10\% FHA and MTA-15\% FHA groups $(P=0.001)$. In the BIA test too, the greatest differences were observed between the MTA and MTA-15\% FHA groups $(P<0.001)$, followed by the MTA and MTA-10\% FHA groups $(P=0.001)$.

Figures 3 and 4 show the linear comparison of the groups and time intervals in the DCA and BIA groups.

According to Figure 3, which shows the linear graph of the DCA test in the three study groups, microbial biofilm formation increased over time in all these groups. The MTA$15 \%$ FHA group exhibited the least microbial biofilms, and the MTA group exhibited the highest microbial biofilm formation at 24- and 72-hour intervals.

According to Figure 4, which shows the liner graph of the BIA test in the three study groups at 24- and 72-hour intervals, the microbial biofilm formation in the MTA-15\% FHA group was less than the two other groups. Microbial 


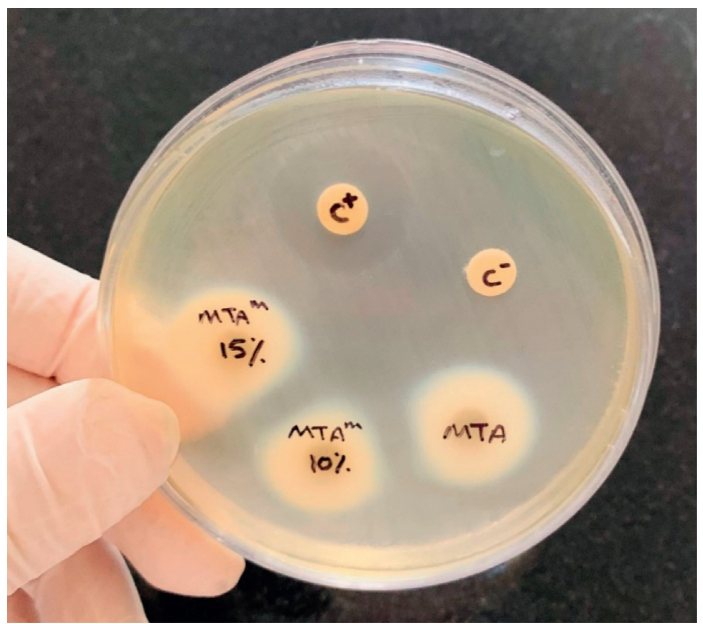

FIGURE 2: Growth inhibition zones in the samples after 24 hours (positive control, $0.2 \%$ CHX; negative control, water).

TABle 1: The means and standard deviations of colony counts $(\mathrm{CFU} / \mathrm{mL})$ based on the DCA test in the study groups.

\begin{tabular}{lccccccccc}
\hline \multirow{2}{*}{ Microbial test } & \multirow{2}{*}{ Groups } & \multicolumn{3}{c}{ After 24 hours } & \multicolumn{4}{c}{ After 72 hours } \\
& & Mean & SD & Min & Max & Mean & SD & Min & Max \\
\hline \multirow{3}{*}{ DCA } & MTA & $30 \times 10^{8}$ & $2.64 \times 10^{8}$ & $28 \times 10^{8}$ & $33 \times 10^{8}$ & $39.66 \times 10^{8}$ & $3.05 \times 10^{8}$ & $37 \times 10^{8}$ & $43 \times 10^{8}$ \\
& MTA-10\% FHA & $22.66 \times 10^{8}$ & $2.08 \times 10^{8}$ & $21 \times 10^{8}$ & $25 \times 10^{8}$ & $32.33 \times 10^{8}$ & $3.21 \times 10^{8}$ & $30 \times 10^{8}$ & $36 \times 10^{8}$ \\
& MTA-15\% FHA & $16.33 \times 10^{8}$ & $2.08 \times 10^{8}$ & $14 \times 10^{8}$ & $18 \times 10^{8}$ & $22.33 \times 10^{8}$ & $3.05 \times 10^{8}$ & $19 \times 10^{8}$ & $25 \times 10^{8}$ \\
\hline
\end{tabular}

TABLE 2: The means and standard deviations of colony counts (CFU/mL) based on the BIA test in the study groups.

\begin{tabular}{lccccccccc}
\hline \multirow{2}{*}{ Microbial test } & \multirow{2}{*}{ Groups } & \multicolumn{3}{c}{ After 24 hours } & \multicolumn{4}{c}{ After 72 hours } \\
& & Mean & SD & Min & Max & Mean & SD & Min & Max \\
\hline \multirow{3}{*}{ BIA } & MTA & $28.33 \times 10^{4}$ & $2.51 \times 10^{4}$ & $26 \times 10^{4}$ & $31 \times 10^{4}$ & $41 \times 10^{4}$ & $4.58 \times 10^{4}$ & $36 \times 10^{4}$ & $45 \times 10^{4}$ \\
& MTA-10\% FHA & $26 \times 10^{4}$ & $1.73 \times 10^{4}$ & $24 \times 10^{4}$ & $27 \times 10^{4}$ & $29 \times 10^{4}$ & $2.64 \times 10^{4}$ & $26 \times 10^{4}$ & $31 \times 10^{4}$ \\
& MTA-15\% FHA & $21 \times 10^{4}$ & $2.64 \times 10^{4}$ & $18 \times 10^{4}$ & $23 \times 10^{4}$ & $23.33 \times 10^{4}$ & $3.51 \times 10^{4}$ & $20 \times 10^{4}$ & $27 \times 10^{4}$ \\
\hline
\end{tabular}

TABLE 3: Comparison of the study groups based on the DCA and BIA tests.

\begin{tabular}{lcccccc}
\hline \multicolumn{2}{c}{ Groups } & M.D & $P$ value & LB \\
\hline \multirow{3}{*}{ Antimicrobial tests } & MTA & MTA-10\% FHA & $7.33 \times 10^{8}$ & 0.001 & $3.13 \times 10^{8}$ & $11.53 \times 10^{8}$ \\
& & MTA-15\% FHA & $15.50 \times 10^{8}$ & $<0.001$ & $11.29 \times 10^{8}$ & $19.70 \times 10^{8}$ \\
& MTA-10\% FHA & MTA-15\% FHA & $8.16 \times 10^{8}$ & 0.001 & $3.96 \times 10^{8}$ & $12.36 \times 10^{8}$ \\
BIA & MTA & MTA-10\% FHA & $7.16 \times 10^{4}$ & 0.004 & $2.43 \times 10^{4}$ & $11.90 \times 10^{4}$ \\
& & MTA-15\% FHA & $12.50 \times 10^{4}$ & $<0.001$ & $7.76 \times 10^{4}$ & $17.23 \times 10^{4}$ \\
& MTA-10\% FHA & MTA-15\% FHA & $5.33 \times 10^{4}$ & 0.027 & $0.59 \times 10^{4}$ & $10.06 \times 10^{4}$ \\
\hline
\end{tabular}

biofilm formation increased over time in all the three groups, with a steeper increase in the MTA group than in the two other groups.

\section{Discussion}

After root canal obturation, a proper seal is necessary to ensure root canal treatment success, especially after eliminating E. faecalis and cleaning the root canal with chemomechanical methods [1]. Although various materials are used to obturate the root canals, none is absolutely ideal [31], and many materials might not provide a hermetic seal.
Therefore, it has been suggested that these materials should prevent bacterial growth [32] and have antibacterial properties.

Several studies have investigated the antimicrobial activity of MTA against E. faecalis, with contradictory results. For example, Prathia et al. (2019) showed that MTA has a better antimicrobial effect on this microorganism than calcium hydroxide sealer [17]. In a study by Usman et al. (2017), using the direct contact test, the MTA sealer exhibited better antimicrobial activity than a bioceramic sealer seven days after mixing [1]. However, Kim et al. (2015) reported that MTA Angelus and ProRoot MTA could not 

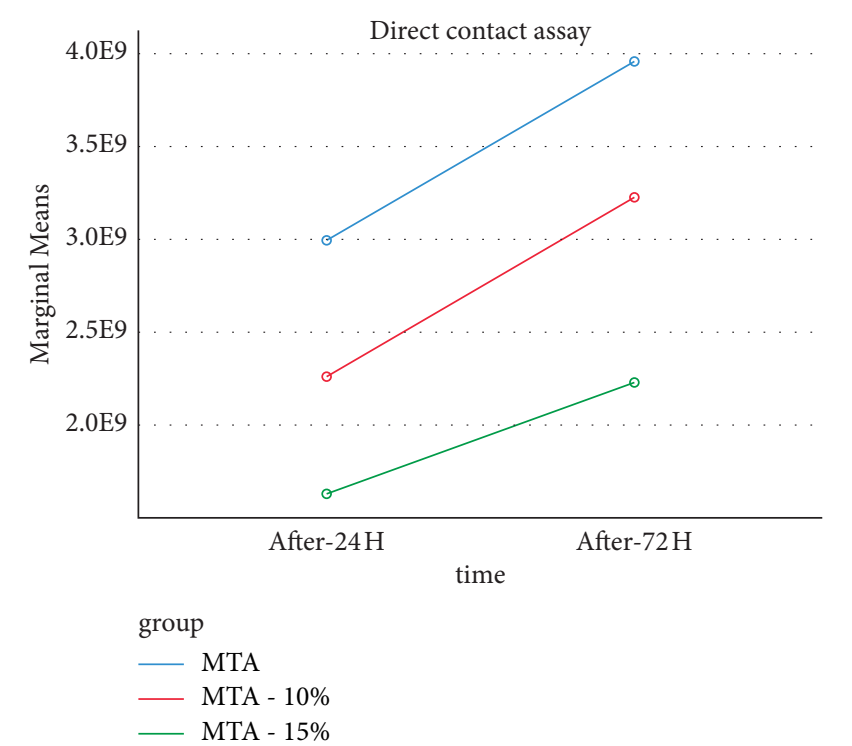

FIGURE 3: The linear graph of the comparison of the groups with the DCA test.

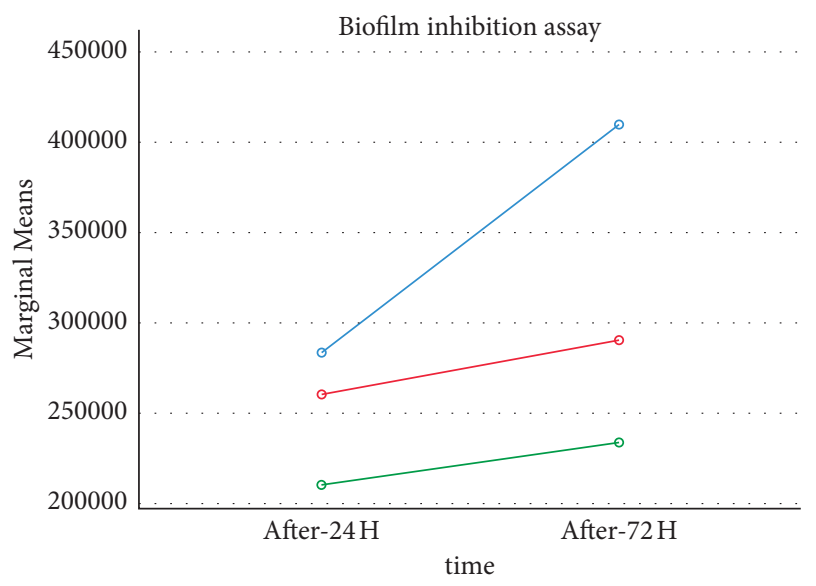

$$
\begin{aligned}
& \text { group } \\
& \text { - MTA } \\
& \text { - } \text { MTA - 10\% } \\
& \text { - } \text { MTA - } 15 \%
\end{aligned}
$$

FIGURE 4: The linear graph of the comparison of the groups with the BIA test.

prevent the growth of E. faecalis [7]. Prathia et al. (2019) and Usman et al. (2017) used the DCA test. Kim et al. (2015) used the DAD test. In the present study, we used DAD, BIA, and DCA tests to evaluate antibacterial properties of the materials. Some studies have hypothesized that MTA releases $\mathrm{Ca}(\mathrm{OH})_{2}$ upon contact with tissue fluid. Therefore, a high $\mathrm{pH}$ value results in continuous antimicrobial activity up to seven days after mixing [17, 33, 34]. However, some other reports have reported that the antibacterial activity cannot be explained only based on the $\mathrm{pH}$ value [7] because, under clinical conditions, the high $\mathrm{pH}$ value of MTA cannot be maintained due to the buffering capacity of dentin [7]. In addition, E. faecalis has a proton pump that helps decrease intracellular $\mathrm{pH}$ [35]. In the present study, no growth inhibition zones were detected after 24 and 72 hours, which might be related to the above explanation.

To improve the antibacterial activity of MTA against E. faecalis, researchers have investigated incorporating various materials into its structure, including $0.2 \% \mathrm{CHX}$, tetracycline, and calcium hydroxide [14]. However, the effect of adding FHA to antimicrobial properties of MTA has not been studied yet. Since there is no similar study to compare the results, further studies are needed.

Only one study evaluated the effect of incorporating this material (nano-FHA) on the antimicrobial activity of the AH26 epoxy resin sealer against E. faecalis and Streptococcus mitis, with the results indicating its positive antibacterial effect compared to the control group [36].

In the present study too, incorporating this material into MTA positively affected its antibacterial effects on E. faecalis. After 24 and 72 hours, the microbial colony counts in the groups modified with nano-FHA were significantly lower than those in the pure MTA group. An increase in the concentration of nano-FHA improved its antibacterial activity.

Wang et al. (2017) carried out a review and described different mechanisms for the antibacterial properties of nanoparticles [37], including oxidative stress induction, the release of metallic ions, and nonoxidative mechanisms [37]. On the other hand, E. faecalis is a Gram-positive bacterial species, and many studies have shown that nanoparticles exhibit greater antimicrobial activity against Gram-positive bacteria than Gram-negative bacteria [37] because the cell wall of Gram-positive bacteria consists of a thin layer of peptidoglycan and teichoic acid, with many pores allowing the penetration of extrinsic molecules, resulting in cell membrane damage and cellular death [37]. In addition, Gram-positive bacteria have a high negative charge on their cell wall that can adsorb nanoparticles [38].

In addition to the mechanisms mentioned above for the antibacterial properties of nanoparticles, fluoride can disrupt the metabolism and growth of oral bacteria directly and indirectly through other complex mechanisms too [39], including disrupting the glycolytic pathway by inhibiting enolase and metalloenzyme, direct inhibition of H+/APPase, decreasing cellular content of peptidoglycans, and interfering with the synthesis of glycogen [39-41]. The results of the studies above might explain the results of the present study.

\section{Conclusion}

The incorporation of nano-FHA into MTA increased its antimicrobial activity against $E$. faecalis compared to pure MTA. Incorporating 10 and 15 wt\% of FHA into MTA significantly increased its antimicrobial activity. In addition, the antimicrobial activity was higher at the 72 -hour interval compared to the 24-hour interval. The highest antibacterial activity was observed after incorporating $15 \mathrm{wt} \%$ of FHA into MTA at the 72-hour interval. It is suggested that future studies evaluate other properties and incorporation of other weight percentages of nanoparticles into MTA. 


\section{Data Availability}

The data used to support the findings of this study are included within the article and are available from the corresponding author upon request.

\section{Conflicts of Interest}

The authors declare that they have no conflicts of interest.

\section{References}

[1] M. Usman, R. Meidyawati, and E. Suprastiwi, "Antibacterial effects of Bioceramic and mineral trioxide aggregate sealers against Enterococcus faecalis clinical isolates," Journal of International Dental and Medical Research, vol. 10, no. 3, pp. 981-986, 2017.

[2] A. F. Fouad, J. Zerella, J. Barry, and L. S. Spångberg, "Molecular detection of Enterococcus species in root canals of therapy-resistant endodontic infections," Oral Surgery, Oral Medicine, Oral Pathology, Oral Radiology \& Endodontics, vol. 99, no. 1, pp. 112-118, 2005.

[3] C. R. Sipert, R. P. Hussne, C. K. Nishiyama, and S. A. Torres, "In vitro antimicrobial activity of fill canal, sealapex, mineral trioxide aggregate, Portland cement and endorez," International Endodontic Journal, vol. 38, no. 8, pp. 539-543, 2005.

[4] L. Grossman, "Antimicrobial effect of root canal cements," Journal of Endodontics, vol. 6, no. 6, pp. 594-597, 1980.

[5] M. Torabinejad, T. F. Watson, and T. R. Pitt Ford, "Sealing ability of a mineral trioxide aggregate when used as a root end filling material," Journal of Endodontics, vol. 19, no. 12, pp. 591-595, 1993.

[6] J. Camilleri, "The chemical composition of mineral trioxide aggregate," Journal of Conservative Dentistry, vol. 11, no. 4, pp. 141-143, 2008.

[7] R. J.-Y. Kim, M.-O. Kim, K.-S. Lee, D.-Y. Lee, and J.-H. Shin, "An in vitro evaluation of the antibacterial properties of three mineral trioxide aggregate (MTA) against five oral bacteria," Archives of Oral Biology, vol. 60, no. 10, pp. 1497-1502, 2015.

[8] R. S. Schwartz, M. Mauger, D. J. Clement, and W. A. Walker, "Mineral trioxide aggregate: a new material for endodontics," The Journal of the American Dental Association, vol. 130, no. 7, pp. 967-975, 1999.

[9] M. Parirokh and M. Torabinejad, "Mineral trioxide aggregate: a comprehensive literature review-Part III: clinical applications, drawbacks, and mechanism of action," Journal of Endodontics, vol. 36, no. 3, pp. 400-413, 2010.

[10] C. Estrela, L. L. Bammann, C. R. Estrela, R. S. Silva, and J. D. Pecora, "Antimicrobial and chemical study of MTA, Portland cement, calcium hydroxide paste," Sealapex and Dycal, vol. 11, 2000.

[11] R. Holland, V. Souza, M. J. Nery, I. M. Faraco Júnior, P. F. E. Bernabé, and J. A. Otoboni Filho, "Reaction of rat connective tissue to implanted dentin tubes filled with a white mineral trioxide aggregate," Brazilian Dental Journal, vol. 13, pp. 23-26, 2002.

[12] S. Alnazhan and A. Aljudai, "Evaluation of antifungal activity of mineral trioxide aggregate," Journal of Endodontics, vol. 29, no. 12 , pp. 826-827, 2003.

[13] K. Al-Hezaimi, K. Al-Hamdan, J. Naghshbandi, S. Oglesby, J. H. S. Simon, and I. Rotstein, "Effect of white-colored mineral trioxide aggregate in different concentrations on Candida albicans in vitro," Journal of Endodontics, vol. 31, no. 9, pp. 684-686, 2005.
[14] N. Gupta, N. Singh, and B. Thapar, "Effect of addition of $2 \%$ chlorhexidine gluconate, calcium hydroxide, and tetracycline powder on antimicrobial activity of mineral trioxide aggregate," Endodontology, vol. 28, no. 1, p. 23, 2016.

[15] V. Peciuliene, I. Balciuniene, H. Eriksen, and M. Haapasalo, "Isolation of Enterococcus faecalis in previously root-filled canals in a Lithuanian population," Journal of Endodontics, vol. 26, no. 10, pp. 593-595, 2000.

[16] V. Peciuliene, A. H. Reynaud, I. Balciuniene, and M. Haapasalo, "Isolation of yeasts and enteric bacteria in rootfilled teeth with chronic apical periodontitis," International Endodontic Journal, vol. 34, no. 6, pp. 429-434, 2001.

[17] T. Prathita, N. K. Djauharie, and R. Meidyawati, "Antimicrobial activity of mineral trioxide aggregate and calcium hydroxide sealer on enterococcus faecalis strain ATCC29212," International Journal of Applied Pharmaceutics, vol. 11, pp. 123-125, 2019.

[18] D. Figdor, J. K. Davies, and G. Sundqvist, "Starvation survival, growth and recovery of Enterococcus faecalis in human serum," Oral Microbiology and Immunology, vol. 18, no. 4, pp. 234-239, 2003.

[19] M. Haapasalo and D. Ørstavik, "In vitro infection and of dentinal tubules," Journal of Dental Research, vol. 66, no. 8, pp. 1375-1379, 1987.

[20] R. D. Morgental, F. V. Vier-Pelisser, S. D. Oliveira, F. C. Antunes, D. M. Cogo, and P. M. P. Kopper, "Antibacterial activity of two MTA-based root canal sealers," International Endodontic Journal, vol. 44, no. 12, pp. 1128-1133, 2011.

[21] G. Verma, K. C. Barick, N. Manoj, A. K. Sahu, and P. A. Hassan, "Rod-like micelle templated synthesis of porous hydroxyapatite," Ceramics International, vol. 39, no. 8, pp. 8995-9002, 2013.

[22] N. Neelakandeswari, G. Sangami, and N. Dharmaraj, "Preparation and characterization of nanostructured hydroxyapatite using a biomaterial," Synthesis and Reactivity in Inorganic Metal-Organic and Nano-Metal Chemistry, vol. 41, no. 5, pp. 513-516, 2011.

[23] A. K. Nayak, "Hydroxyapatite synthesis methodologies: an overview," International Journal of Chem Tech Research, vol. 2, no. 2, pp. 903-907, 2010.

[24] C. Robinson, R. C. Shore, S. J. Brookes, S. Strafford, S. R. Wood, and J. Kirkham, "The chemistry of enamel caries," Critical Reviews in Oral Biology \& Medicine, vol. 11, no. 4, pp. 481-495, 2000.

[25] X. Ge, Y. Leng, C. Bao, S. L. Xu, R. Wang, and F. Ren, "Antibacterial coatings of fluoridated hydroxyapatite for percutaneous implants," Journal of Biomedical Materials Research Part A, vol. 95A, no. 2, pp. 588-599, 2010.

[26] K. Shekofteh, A. Boruziniat, M.-J. Moghaddas, F. Namdar, E. Zahabi, and H. Bagheri, "Formulation and mechanical characterization of a semi-crystalline nano-fluorine hydroxyapatite-filled dental adhesive," Journal of the Australian Ceramic Society, vol. 54, no. 4, pp. 731-738, 2018.

[27] M. A. Wikler, "Methods for dilution antimicrobial susceptibility tests for bacteria that grow aerobically: approved standard," CLSI (NCCLS), vol. 26, 2006.

[28] A. Sodagar, A. Akhavan, S. Arab, A. Bahador, M. Pourhajibagher, and A. Soudi, "Evaluation of the effect of propolis nanoparticles on antimicrobial properties and shear bond strength of orthodontic composite bonded to bovine enamel," Frontiers in dentistry, vol. 16, no. 2, pp. 96-104, 2019.

[29] M. Pourhajibagher, L. Ranjbar Omrani, M. Noroozian, Z. Ghorbanzadeh, and A. Bahador, "In vitro antibacterial 
activity and durability of a nano-curcumin-containing pulp capping agent combined with antimicrobial photodynamic therapy," Photodiagnosis and Photodynamic Therapy, vol. 33, p. 102150, 2021.

[30] E. Gholibegloo, A. Karbasi, M. Pourhajibagher et al., "Carnosine-graphene oxide conjugates decorated with hydroxyapatite as promising nanocarrier for ICG loading with enhanced antibacterial effects in photodynamic therapy against Streptococcus mutans," Journal of Photochemistry and Photobiology B: Biology, vol. 181, pp. 14-22, 2018.

[31] M. Hasan Zarrabi, M. Javidi, M. Naderinasab, and M. Gharechahi, "Comparative evaluation of antimicrobial activity of three cements: new endodontic cement (NEC), mineral trioxide aggregate (MTA) and Portland," Journal of Oral Science, vol. 51, no. 3, pp. 437-442, 2009.

[32] M. Torabinejad, C. U. Hong, T. R. P. Ford, and J. D. Kettering, "Antibacterial effects of some root end filling materials," Journal of Endodontics, vol. 21, no. 8, pp. 403-406, 1995.

[33] S. R. Sluyk, P. C. Moon, and G. R. Hartwell, "Evaluation of setting properties and retention characteristics of mineral trioxide aggregate when used as a furcation perforation repair material," Journal of Endodontics, vol. 24, no. 11, pp. 768-771, 1998.

[34] T. J. Stowe, C. M. Sedgley, B. Stowe, and J. C. Fenno, "The effects of chlorhexidine gluconate $(0.12 \%)$ on the antimicrobial properties of tooth-colored ProRoot mineral trioxide aggregate," Journal of Endodontics, vol. 30, no. 6, pp. 429-431, 2004.

[35] C. Stuart, S. Schwartz, T. Beeson, and C. Owatz, "Enterococcus faecalis: its role in root canal treatment failure and current concepts in retreatment," Journal of Endodontics, vol. 32, no. 2, pp. 93-98, 2006.

[36] B. A. Jerri Al-Bakhsh, F. Shafiei, M. Pourhajibagher, K. Shekofteh, A. Hashemian, and M. Behroozibakhsh, "The antibacterial activity of an epoxy resin-based dental sealer containing bioactive glass, hydroxyapatite, and fluorohydroxyapatite nanoparticles against Enterococcus Faecalis and Streptococcus mitis," Nanomedicine Journal, vol. 7, no. 1, pp. 13-20, 2020.

[37] L. Wang, C. Hu, and L. Shao, "The antimicrobial activity of nanoparticles: present situation and prospects for the future," International Journal of Nanomedicine, vol. 12, pp. 1227-1249, 2017.

[38] A. Sarwar, H. Katas, S. N. Samsudin, and N. M. Zin, "Regioselective sequential modification of chitosan via azidealkyne click reaction: synthesis, characterization, and antimicrobial activity of chitosan derivatives and nanoparticles," PLoS One, vol. 10, no. 4, p. e0123084, 2015.

[39] I. Hamilton, "Biochemical effects of fluoride on oral bacteria," Journal of Dental Research, vol. 69, no. 2, pp. 660-667, 1990.

[40] A. Alhilou, T. Do, L. Mizban, B. H. Clarkson, D. J. Wood, and M. G. Katsikogianni, "Physicochemical and antibacterial characterization of a novel fluorapatite coating," ACS Omega, vol. 1, no. 2, pp. 264-276, 2016.

[41] L. Wang, S. He, X. Wu et al., "Polyetheretherketone/nanofluorohydroxyapatite composite with antimicrobial activity and osseointegration properties," Biomaterials, vol. 35, no. 25, pp. 6758-6775, 2014. 